\title{
Campus Event App - New Exploration for Mobile Augmented Reality
}

\author{
Joseph T. Chao and Lei Pan \\ Bowling Green State University \\ Bowling Green, Ohio, USA
}

\section{jchao@bgsu.edu leipan@bgsu.edu}

\author{
Kevin R. Parker \\ Idaho State University \\ Pocatello, Idaho, USA
}

parkerkr@isu.edu

\begin{abstract}
Augmented Reality (AR) enhances the real world environment with virtual information by embedding virtual objects in the real world to enhance the user's life experience. Although AR technology has been around for fifty years, it has not been widely applied in practice until recent years when mobile phone technologies became more mature. Researchers have started to investigate the combination of AR and mobile phone technology - mobile AR - due the popularity of mobile devices. Mobile AR is a new and expanding area, and there are many research opportunities in the mobile AR field. The purpose of this project is to build a mobile AR application - the Campus Event App - as a means of demonstrating how mobile AR technologies can be used in combination with other applications to produce a useful and practical tool. The app brings new user experiences to event searching based on a combination of AR, mobile technology, and global positioning system (GPS) location-based techniques. The application is intended to help users to find daily campus events by visualizing events over the real world and showing the map and route to the event in real time.
\end{abstract}

Keywords: Augmented Reality, Mobile App, GPS, Location-based, Visualization, Campus Event, Android SDK, Metaio SDK, Google Geocoding.

\section{Introduction}

Augmented Reality (AR) augments the real world environment with virtual information such as sound, video, graphics or global positioning system (GPS) data to enhance life experiences. AR falls between the real-world environment and a Virtual Environment. Although AR may be considered to be a variation of Virtual Reality (VR), there are significant differences. AR integrates virtual objects into the real environment, allowing users to see both real world and virtual objects, whereas VR immerses users in a completely virtual environment. In short, AR enhances reality

Material published as part of this publication, either on-line or in print, is copyrighted by the Informing Science Institute. Permission to make digital or paper copy of part or all of these works for personal or classroom use is granted without fee provided that the copies are not made or distributed for profit or commercial advantage AND that copies 1) bear this notice in full and 2) give the full citation on the first page. It is permissible to abstract these works so long as credit is given. To copy in all other cases or to republish or to post on a server or to redistribute to lists requires specific permission and payment of a fee. Contact Publisher@InformingScience.org to request redistribution permission. by placing virtual objects in the real environment.

AR technology can be applied to many areas including military, medical, education, and tourism. The heads-up display (HUD) is an AR application in the military. The head-mounted display (HMD) can display critical data such as enemy locations in a soldier's line of sight (Azuma, 1997). Doctors can use AR 
technology to practice surgery in a controlled virtual environment. AR applications have been used in education as well (Mountney, Giannarou, Elson, \& Yang, 2009). Textbooks, flashcards and other educational material can include markers so that when they are scanned by an AR device, they can produce additional information such as video clips, images, or other supplemental information to readers (Hanna, 2012). In terms of tourism applications, AR can enhance tourists' experience when travelling by offering additional real-time information such as location, features, or comments regarding tourist attractions.

Although the term augmented reality was coined in 1990 by Thomas Caudell, a Boeing researcher, AR has been around for a long time. In 1962, a simulator called Sensorama with visuals, sound, vibration, and smell was developed and patented by Morton Heilig (1962). In 1968, the first head-mounted display was created by Ivan Sutherland (1968). In 1975, Videoplace was developed by Myron Krueger (1985) to allow users to interact with virtual objects. By the 1990s, some companies were using AR technology for training, visualization, and other applications. In this decade AR technology has been driven by the evolution of wireless technology that enables users to experience AR in personal computers and mobile devices. AR-based mobile applications such as AR mapping and social tools have begun to appear on the market in recent years. ARbased mobile applications are attracting more and more attention since the combination of AR technology and mobile technology is very powerful and the applications can revolutionize the user experience in many areas. With mobile technology, it's much easier to apply AR technology to people's daily life than even before.

The purpose of this project is to build a mobile AR application as a means of demonstrating how mobile AR technologies can be used in combination with other applications to produce a useful and practical tool. Thus, this study involves conceptual development that is demonstrated by a fully functioning campus event mobile app. The Campus Event App is an example of the combination of AR technology and mobile technology. The application can bring new user experiences to the field of event searching applications. Event searching focuses on helping people find and attend events that match their interests. The problem that the app was designed to address dealt with the fact that the multitude of campus events at a university every day makes it challenging for students to be aware of all the interesting events near or at the current location. Further, it would be helpful if they had access to a map and the route to the event in real time. Most of mobile event applications that are currently available can only help users to find physical addresses of events. Campus Event App is a GPS location-based AR mobile application that can help users to find scheduled events near the current location by visualizing the events over a real scene and displaying a map and route in real time. The application is developed based on the Android software development kit (SDK) and the Metaio SDK. The architecture of the application is a clientserver structure. A database is built for the application, and the Google Geocoding application

programming interface (API) is used to address some difficult problems in the development of the application.

\section{Literature Review}

Recent studies have investigated the combination of AR and mobile technologies and proposed various approaches to and algorithms for the mobile AR field. Of particular relevance to this project are the studies that include user location tracking and markerless tracking of AR components. Markerless AR is gradually replacing fiducial marker-based AR. Fiducial marker-based AR systems required that target objects contain artificial information (fiducial markers) to enable tracking. Markerless AR relies on natural features of reality that can be directly extracted from characteristics in the environment. 
Hence, this section will examine studies that focus on location tracking of users' current locations, accurate and quick tracking of points of interests, outdoor tracking of points of interests, and markerless tracking algorithms for mobile AR.

User location tracking is a prerequisite for location-based applications. In order for the Campus Event App to provide relevant event information according to users' locations, the app needs accurate position tracking to display AR components such as points of interests in users' real environment. Various researches have been done regarding user location tracking for both outdoor and indoor position tracking. In terms of outdoor location tracking, GPS is capable of providing global position that has accuracy range from centimeters to several meters. Since the accuracy largely depends on good signals from multiple satellites, GPS tracking may not work well at some places such as locations inside a building where devices do not receive good signals (Panzieri, Pascucci, \& Ulivi, 2002). Researchers considered other approaches to improve indoor location tracking rather than relying on GPS signals. Kyamakya, Zapater, and Lue (2003) proposed an approach using Bluetooth, available on most modern mobile devices, to detect indoor locations. Another system called Bat system which is demonstrated by Hazas and Hopper (2006) is based on the Ultrasounds technology. In this system, a user wears a badge that emits an ultrasonic pulse when a central controller triggers the radio. The system can determine the time of pulses flying from badges to the network receivers, and then calculate the 3D position based on a multilateration algorithm. Radio Frequency Identification (RFID) has also been used in the research of indoor position tracking. The estimation of RFID position is based on communication between RFID readers and tags. The nature of RFID fits well with indoor position tracking (Bekkali, Sanson, \& Matsumoto, 2007). Ozdenizci, Ok, Coskun, and \& Aydin (2011) developed indoor tracking system using Near Field Communication (NFC) which is able to read the tags. NFC is developed based on RFID technology . Lee and Mase (2001) proposed another approach which uses local sensors and dead reckoning techniques to compute users' current locations.

Accurately and quickly detecting and tracking points of interest is another important feature for developing good AR mobile applications. Langlotz et al. (2011) demonstrates several techniques to improve the accuracy of positions of points of interest in a panoramic image surrounding a mobile user. Even when faced with changing surroundings, they were able to attain an accurate match rate of $90 \%$ testing the system in real time using a mobile phone. Jo, Hwang, Park, and Ryu (2011) present a focus +context visualization method to help find points of interest in an AR mobile application. They compare the method with top-down 2D radar and 3D arrow visualization approaches to evaluate their method. Wientapper, Wuest, and Kuijper (2011) propose a tracking approach that provides quick and accurate frame-by-frame tracking by combining the techniques of initializing tracking and obtaining scene geometry.

Outdoor tracking of points of interest is critical for mobile AR, but it is quite difficult. Various studies detail experiments and approaches to improve outdoor tracking of AR components. Taketomi, Sato, and Yokoya (2011) present a two-stage outdoor tracking method. In the first step, they construct a landmark database based on motion data and laser range finder information. In the second step, landmark tracking is used to provide outdoor tracking for AR mobile application. Wither, Tsai, and Azuma (2011) describe an approach that they call "Indirect Augmented Reality" to get good AR mobile experiences by using tracking information from mobile phones in outdoor environments. They align augmentations with pre-recorded static panoramic images and find that users consider this approach to be compelling for some outdoor AR scenarios. Morrison et al. (2011) demonstrate and investigate the benefits of using a physical map for collaborative work and the benefits of using a physical map that is augmented with personal information to assist in navigation. Gee et al. (2011) propose a system that is able to operate both indoors and outdoors by integrating a range of different tracking technologies. They successfully create and visualize large numbers of AR annotations over a range of various locations by integrating global po- 
sitioning from indoor ultra-wideband technology and GPS with real-time visual simultaneous localization and mapping (SLAM).

Markerless tracking is the foundation of tracking points of interest. Some researchers proposed several important algorithms in the field which set up the foundation for further research. Davison, Reid, Molton, and Stasse (2007) describe the MonoSLAM system that is the most widely known work in monocular camera localization mapping. They use a monocular camera as the only input device in order to apply the SLAM method to the vision domain. A sparse map of oriented planar textures is maintained by MonoSLAM. Before updating the map dynamically, the system uses a planar target in order to initialize the system. The result, which reaches $30 \mathrm{~Hz}$ at real time operation when using AR, is presented in the AR domain. Klein and Murray (2007) introduce the Parallel Tracking and Mapping (PTAM) system, which uses a multi-threaded approach that morphs and matches image patches in order to track point of interests simultaneously. The PTAM can successfully determine and update the locations of points of interest by using the bundle adjustment method for visual refinements. Taehee and Hollerer (2009) develop a hybrid tracking method that combines Scale-invariant feature transform (SIFT) and the optic flow tracking method. SIFT, an algorithm applicable to computer vision (Lowe, 1999), detects and describes local features in images. The system extracts and matches SIFT features frequently in a thread that is separate from the tracker to realize real-time performance and scene recognition.

Mobile AR is an evolving field. The Campus Event App combines not only AR technology and mobile technology, but also integrates GPS location-based techniques. The convergence of AR, mobile, and GPS is explored with the expectation of improving the performance of event searching applications and enhancing the user experience.

\section{Campus Event App}

This project was initiated to demonstrate how AR technology could be used to enhance mobile apps. As the concept evolved, it became evident that GPS location-based features would also be required. Recall that the Campus Event App was described above as an event searching tool that could assist users in locating events in their immediate vicinity in real time, but in addition would display a map and the best route to a selected event. It was envisioned as a tool for college students to locate campus or area events, and find the route to the event. While there currently exist tools that could be used in combination to determine such information, it can be cumbersome to use multiple tools for a single task. For example, calendar applications or similar event applications only provide users with physical addresses of events. If users would like to determine the proximity of the events or the route from their current location to the events, they have to enter the address provided by the app into mapping applications such as Google Map to see the distance and the route between them. Campus Event App allows users to see virtual daily events in their vicinity visualized over the reality on the phone screen and check the map and route to the event they pick.

There are multiple user scenarios in the Campus Event App. In Scenario One, after a user starts the application, he or she will see the main menu that contains two options: Augmented Reality and Event List. If the user selects the AR option and then points the camera toward a direction, she will be able to see virtual billboards of nearby events in that direction visualized and placed over the reality. If the user selects Event List then a list of upcoming events is displayed. This option is provided so that even if the GPS or camera doesn't work in the user's phone, they can still use the application. Figure 1 shows an example of the AR view and an event list. 

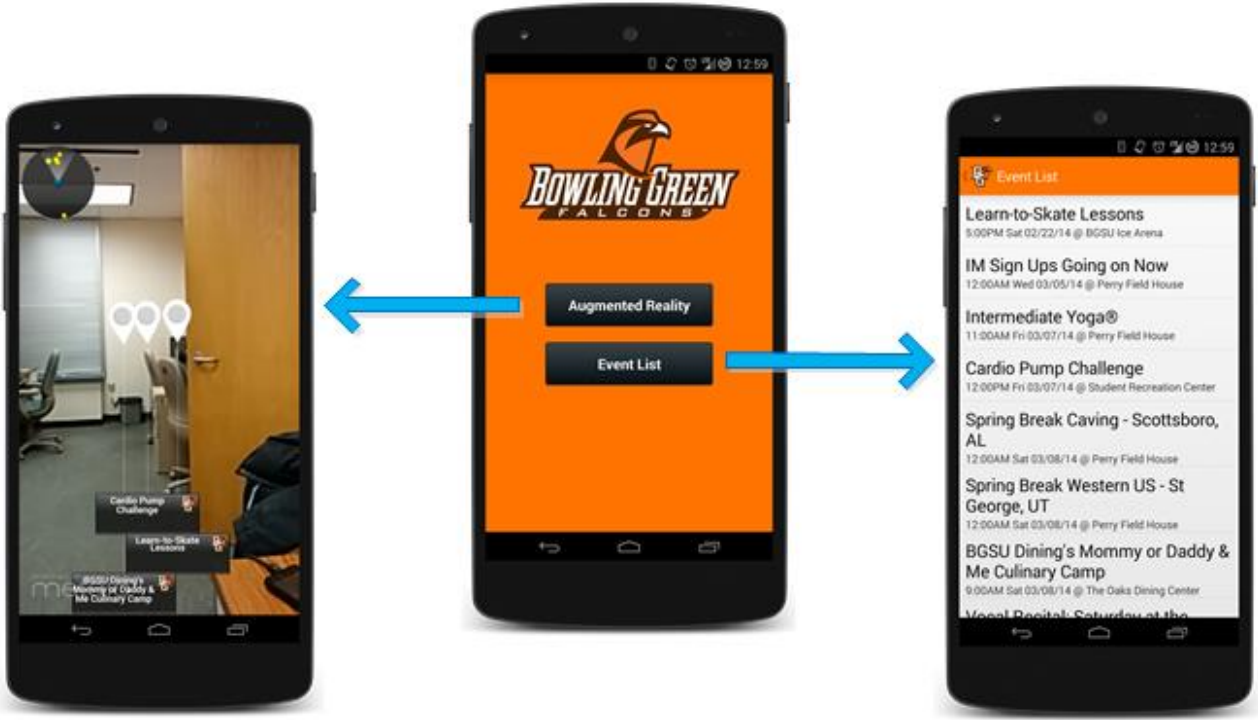

Figure 1. Two main functions of Campus Event App.

In Scenario 2, the user can select an event in either the AR View or from the event list. After the user makes a selection, the system will bring up the event detail screen. The event detail screen provides more information about the event such as the event date and time, the event location, and an event description. The screen also provides an event map button and an event link button. Figure 2 shows an example of the Event Details screen. When the user clicks the Event Link button, she is taken to the web site for the event (if available), and when the Map button is clicked a map with the event location is displayed, and from there the user can also display the route from the current location to the event.

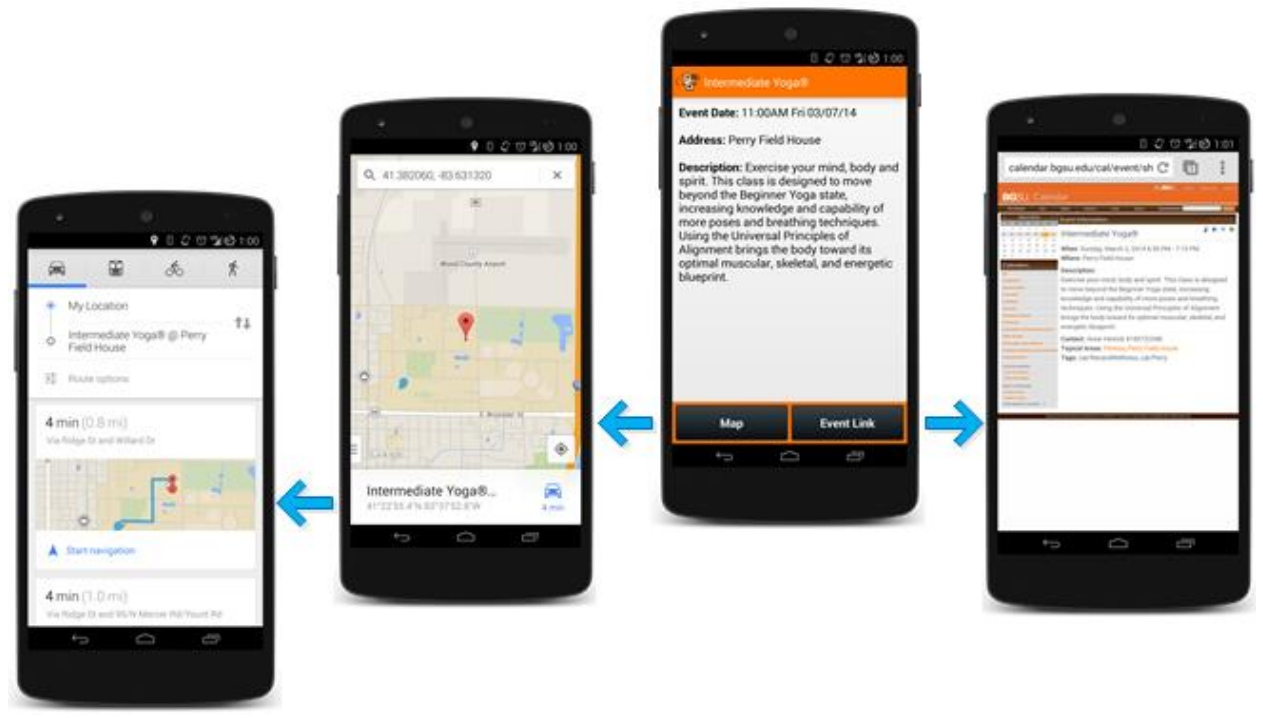

Figure 2: Event Details, Map, and Web Link.

Figure 3 shows multiple AR views in various directions. It also demonstrates that clicking on one of the events brings up the event detail screen. 

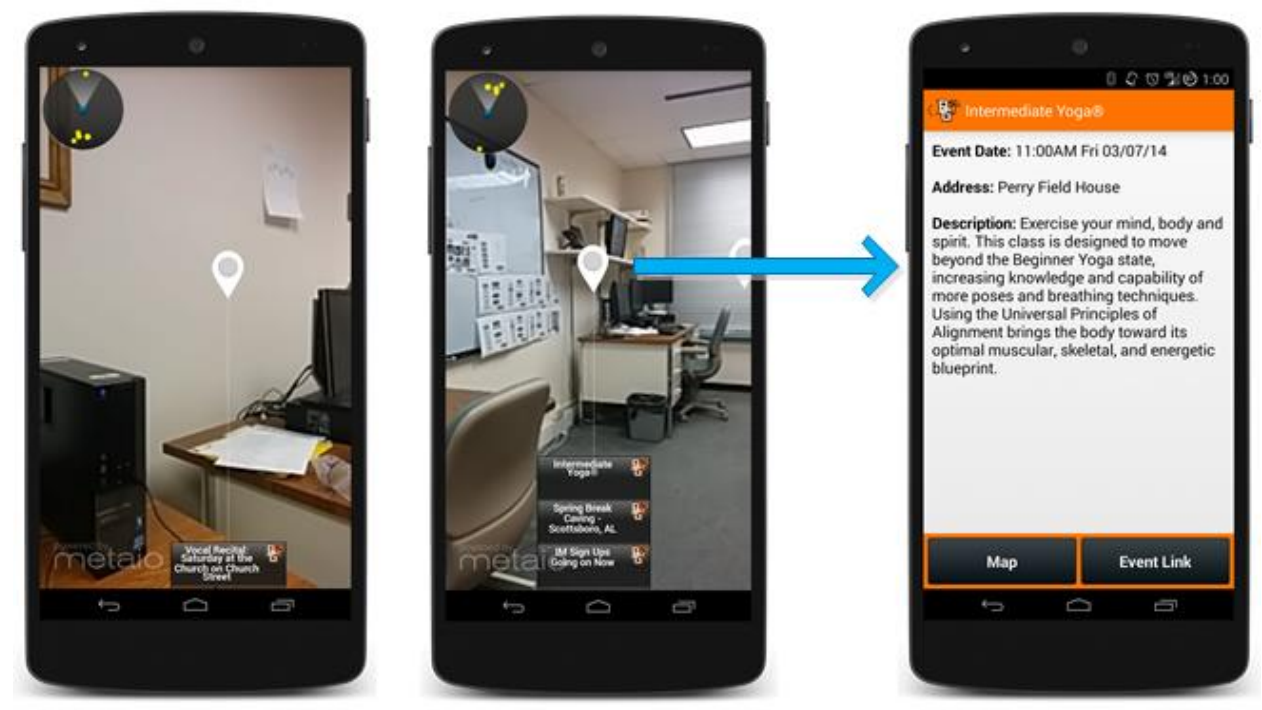

Figure 3: AR views. Clicking an event on $A R$ view takes the user to event detail screen.

\section{Implementation}

\section{Technology}

The Campus Event App requires AR to interact with physical reality through a phone camera. The application needs live data and meta data. Live data can be collected through camera and location data can be collected by using a GPS API. Some SDKs are needed to visualize AR elements over a camera feed in order to bring virtual graphics and a real scene together. Android SDK and Metaio SDK were chosen to develop an AR Android application since the Android SDK provides a large variety of API libraries and developer tools that can be used to build, test, and debug apps for Android applications. The Android bundle was used in the development because it includes the key components of the Android SDK and a version of the Eclipse integrated development environment (IDE) with the Android Developer Tool (ADT), which can be used to speed up Android app development. Metaio SDK was also used because it provides all the libraries for augmented reality application development. Metaio SDK assists with the flexible deployment and licensing of a stand-alone or cloud-based AR app project.

In order to use an SDK to visualize events and align visualized data with pre-recorded static panoramic images accurately on the phone screen, the coordinates of all the addresses of events are needed. There are two steps required to obtain the coordinates of all the addresses. The first step is to fetch the addresses of events near the current location, and the second step is to convert the addresses to longitude, latitude, and altitude (LLA) coordinates. There are several methods to get event addresses. One way is to parse event addresses from daily campus email updates. Since there is no XML feed, JSON feed, or RSS feed for campus email updates, simply parsing email to get addresses is not an effective approach. An alternative is to build a website that allows event organizers or promoters to input event data, such as addresses, following a particular format. By doing so, accurate addresses can be obtained from the website. However, this requires an online central clearinghouse for gathering event data, and a willingness to do so on the part of event organizers. The most feasible alternative for fetching addresses was to access the database from the university calendar website. This approach was selected because the calendar website generates an XML feed, JSON feed, or RSS feed, and it is easier to obtain addresses when they are consistently formatted. In addition, this resource already exists, so no additional time or cost was required. 
Converting physical addresses to LLA coordinates is the second step. The Campus Event App needs longitude, latitude, and altitude (LLA) coordinates instead of physical addresses in order to annotate points of interests accurately in the panoramic scene. There are multiple ways to convert addresses to coordinates. One approach is to build a table into which addresses and corresponding coordinates are inserted, and the application is then able to query and fetch coordinates based on the input addresses. This approach is often used to convert addresses to coordinates because the local database can speed up the application. However, a precondition for using this approach is that the input addresses must exactly match the addresses stored in the database, or exactly match part of the addresses if approximation queries are used. In our case, however, the precondition was not satisfied.

The format of input addresses that the application fetches from the university calendar system doesn't follow specific rules and is often inconsistent. For example, people might input various addresses for the same location, Bowen-Thompson Student Union, such as BTSU or Student Union. If we build a table and store data pairs like "Bowen-Thompson Student Union" "$41.12121212,-81.33442$ ", and then query the lookup table using "BTSU", the query will return no match. In addition, addresses could have data entry errors, in which case queries will again be unsuccessful. Hence, another approach, Google Geocoding, was considered to improve the situation. Google Geocoding has strong approximation search and match algorithms. If we query "BTSU" or something that contains a typo using Google Geocoding, it will return the correct LLA coordinates. However, some adjustments and modifications were required in order to handle some query exceptions from Google Geocoding.

A database that is capable of storing augmentation data such as addresses, coordinates, and other event details is needed. Augmentation data can be stored and accessed through an online database or a local database. An online MySQL database was selected for the application. Local databases were discounted because there can be time and space performance issues with local relational databases. Since a large amount of event information will be input into the database and several queries will interact with the database every day, if local databases are used inside the application, the speed of application will be decreased. Since local data storage consumes space, if a local database is used, the application will require more space to operate efficiently. Thus, the decision was made to use an online database.

\section{Event Data Flow}

The Campus Event App is based on the client-server model. The mobile phone app acts as the client, and an App Server, which is both a server and the server-side software that retrieves event data from the university calendar, stores it in a database, and later provides it for the user when the client device requests it, constitutes the server portion. An analysis of the data flow begins with the App Server obtaining all of the event information, such as addresses, event name, event description, event time, and event links, from the university calendar. Second, the App Server software sends the physical addresses of all the events to Google Geocoding, which generates the LLA coordinates. Third, the App Server software stores all of the event information, including all of the data obtained from the university calendar as well as the LLA obtained from Google Geocoding, into a database on the server. Finally, the Campus Event App on the client device makes a request for data and the App Server processes the request, fetches the desired event data from the database, and sends that data in JavaScript Object Notation (JSON) format to the mobile phone. The data flow for Campus Event App is depicted in Figure 4. 


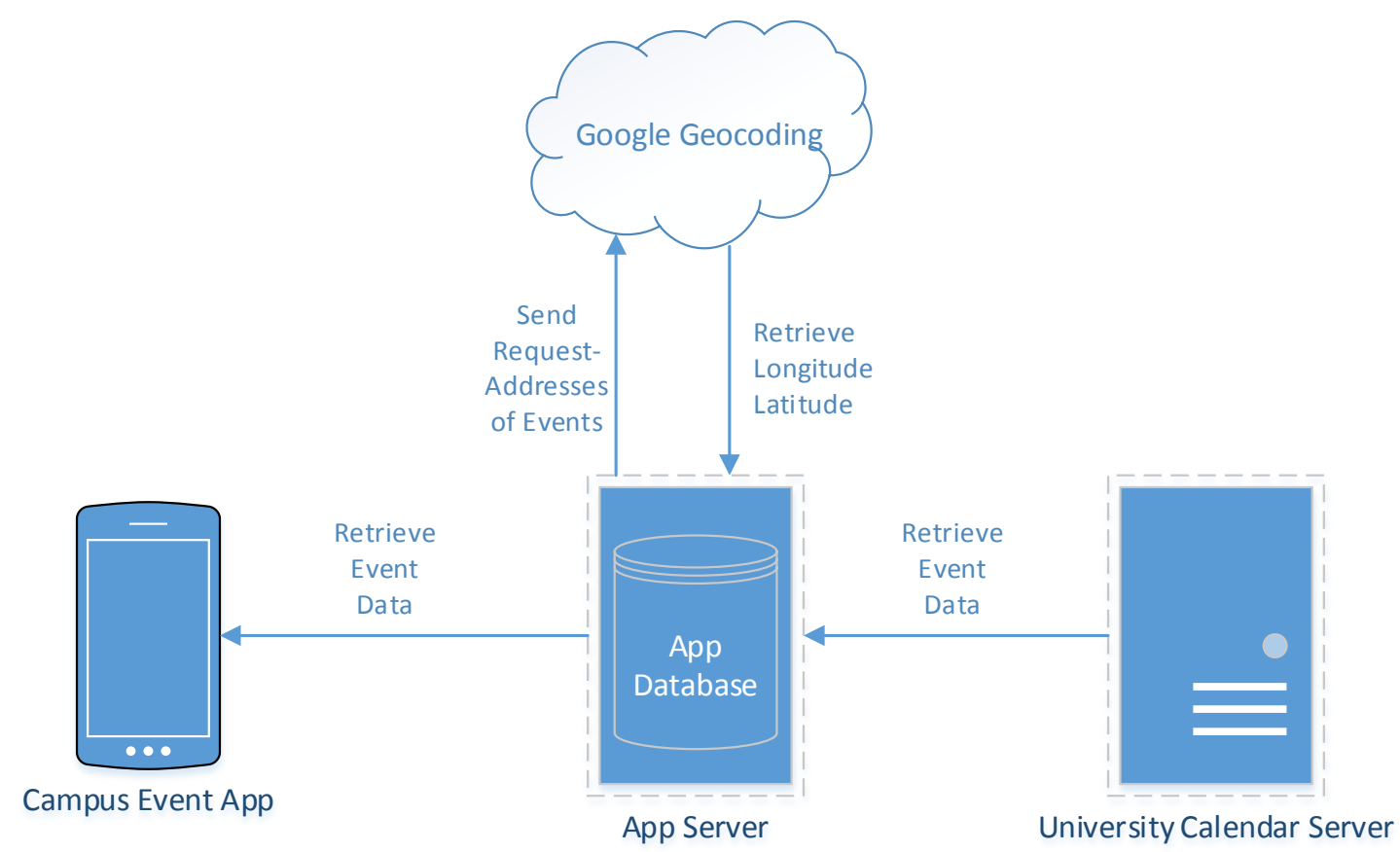

Figure 4: Data Flow through Client and Server.

\section{Future Work}

Multiple features can be added to the Campus Event App in order to improve the interface. When the application visualizes events as virtual billboards on the phone screen, it displays only event names. One enhancement would be to display the distance between the current location and the event location on the virtual billboards. Such a feature would allow users to find the distance of every event from their current location.

Another enhancement might be to provide a filter to categorize the events. There are often many events in a single day, and currently all of them are displayed on the screen. Many users, however, may not be interested in all types of options and may prefer to see only events that match their preferences rather than all the events. A filter that can select only events in selected categories will be useful a useful feature.

Another useful enhancement is a Date Pick option. Currently the application will only show events for the current date. It is not unreasonable for users to want to see upcoming events. The Date Pick feature would allow users to select any date and the application will show corresponding events for that date.

A complementary feature is Add to Calendar. If a user accesses the Date Pick option and finds an upcoming event that he or she would like to attend, an Add to Calendar feature would enable users to add the event to their calendar so they remember to attend. These improvements can improve the Campus Event App and result in an even better user experience.

\section{Conclusion}

Mobile AR is an evolving field with many opportunities for both research and development. This project used app development to explore how diverse technologies like AR, mobile, and locationbased techniques can be melded together into a useful and practical tool. The resulting AR application can help users find interesting events in their vicinity by visualizing event information, bringing new innovations to the event searching field. Further, the application that was developed 
demonstrates not only that AR has practical uses, but also shows how a variety of existing technologies can be combined to bring about a tool that addresses a perceived need for university students by embracing different types of media and applications.

This study makes a contribution by applying existing theory and tools to develop a mobile AR app with real world applications, demonstrating how AR can be used in conjunction with other technologies to build even more useful AR applications. This is an excellent example of technological convergence, that is, the combination of previously separate technologies in such a way that they interact with each other synergistically to enhance human capabilities and serve human needs (Olawuyi \& Mgbole, 2012).

\section{References}

Azuma, R. T. (1997). A survey of augmented reality. Presence: Teleoperators and Virtual Environments, 6(4), 355-385.

Bekkali, A., Sanson, H., \& Matsumoto, M. (2007). RFID indoor positioning based on probabilistic RFID map and Kalman filtering. In Third IEEE International Conference on Wireless and Mobile Computing, Networking and Communications, 2007. WiMOB 2007 (pp. 21-21).

Davison, A. J., Reid, I. D., Molton, N. D., \& Stasse, O. (2007). MonoSLAM: Real-time single camera SLAM. IEEE Transactions on Pattern Analysis and Machine Intelligence, 29(6), 1052-1067.

Gee, A. P., Webb, M., Escamilla-Ambrosio, J., Mayol-Cuevas, W., \& Calway, A. (2011). A topometric system for wide area augmented reality. Computers \& Graphics, 35(4), 854-868.

Hazas, M., \& Hopper, A. (2006). Broadband ultrasonic location systems for improved indoor positioning. IEEE Transactions on Mobile Computing, 5(5), 536-547.

Jo, H., Hwang, S., Park, H., \& Ryu, J. (2011). Mobile augmented reality: Aroundplot: Focus+Context interface for off-screen objects in 3D environments. Computers \& Graphics, 35(4), 841-853.

Klein, G., \& Murray, D. (2007). Parallel tracking and mapping for small AR workspaces. In 6th IEEE and ACM International Symposium on Mixed and Augmented Reality, 2007. ISMAR 2007 (pp. 225-234).

Krueger, M. W., Gionfriddo, T., \& Hinrichsen, K. (1985). VIDEOPLACE - An artificial reality. Proceedings of the SIGCHI Conference on Human Factors in Computing Systems (pp. 35-40).

Kyamakya, K., Zapater, A., \& Lue, Z. (2003). An indoor Bluetooth-based positioning system: Concept, implementation and experimental evaluation. Proceedings of the International Conference on Wireless Networks.

Heilig, Morton L (1962). Sensorama simulator. U.S. Patent No. 3,050,870. Washington, DC: U.S.

Langlotz, T., Degendorfer, C., Mulloni, A., Schall, G., Reitmayr, G., \& Schmalstieg, D. (2011). Robust detection and tracking of annotations for outdoor augmented reality browsing. Computers \& Graphics, $35(4), 831-840$.

Lee, S.-W., \& Mase, K. (2001). Incremental motion-based location recognition. Proceedings of the Fifth International Symposium on Wearable Computers, 2001, (pp. 123-130).

Lee, T., \& Hollerer, T. (2009). Multithreaded hybrid feature tracking for markerless augmented reality. IEEE Transactions on Visualization and Computer Graphics, 15(3), 355-368.

Lowe, D. G. (1999). Object recognition from local scale-invariant features. Proceedings of the Seventh IEEE International Conference on Computer Vision, 1999 (Vol. 2, pp. 1150-1157 vol.2).

Morrison, A., Mulloni, A., Lemmelä, S., Oulasvirta, A., Jacucci, G., Peltonen, P., Schmalstieg D., Regenbrecht, H. (2011). Collaborative use of mobile augmented reality with paper maps. Computers \& Graphics, 35(4), 789-799. 
Mountney, P., Giannarou, S., Elson, D., \& Yang, G.-Z. (2009). Optical biopsy mapping for minimally invasive cancer screening. International Conference on Medical Image Computing and ComputerAssisted Intervention, 12(Pt 1), 483-490.

Olawuyi, J. O., \& Mgbole, F. (2012). Technological convergence. Science Journal of Physics, 2012, Retrieved December 6, 2013 from http://www.sjpub.org/sjp/sjp-221.pdf

Ozdenizci, B., Ok, K., Coskun, V., \& Aydin, M. N. (2011). Development of an indoor navigation system using NFC technology. In 2011 Fourth International Conference on Information and Computing (ICIC) (pp. 11-14).

Stewart-Smith, H. (2012). Education with augmented reality: AR textbooks released in Japan, ZDnet, Retrieved December 8, 2013, from http://www.zdnet.com/blog/asia/education-with-augmented-reality-artextbooks-released-in-japan-video/1541

Panzieri, S., Pascucci, F., \& Ulivi, G. (2002). An outdoor navigation system using GPS and inertial platform. IEEE/ASME Transactions on Mechatronics, 7(2), 134-142.

Sutherland, I. E. (1968). A head-mounted three dimensional display. Proceedings of the December 9-11, 1968, Fall Joint Computer Conference, Part I (pp. 757-764).

Taketomi, T., Sato, T., \& Yokoya, N. (2011). Real-time and accurate extrinsic camera parameter estimation using feature landmark database for augmented reality. Computers \& Graphics, 35(4), 768-777.

Wientapper, F., Wuest, H., \& Kuijper, A. (2011). Composing the feature map retrieval process for robust and ready-to-use monocular tracking. Computers \& Graphics, 35(4), 778-788.

Wither, J., Tsai, Y.-T., \& Azuma, R. (2011). Indirect augmented reality. Computers \& Graphics, 35(4), $810-822$.

\section{Biographies}

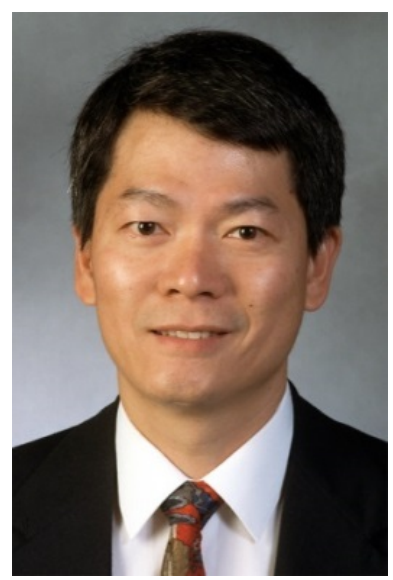

Dr. Joseph T. Chao is an Associate Professor and the Director of the Agile Software Factory in the Department of Computer Science at Bowling Green State University. The Software Factory, which he founded in 2008 with a grant from the Agile Alliance, provides students with service-learning opportunities and real-world project experience in software engineering. Prior to entering academia, Dr. Chao gained more than seven years of industry experience working as Software Engineer, System Analyst, QA Tester, Project Manager as well as Director of Software Development. His research focus is on software engineering with special interests in agile methods, web and mobile technologies, and object-oriented analysis and design. Dr. Chao holds an M.S. in Operations Research from Case Western Reserve University and a Ph.D. in Industrial and Systems Engineering from The Ohio State University. 


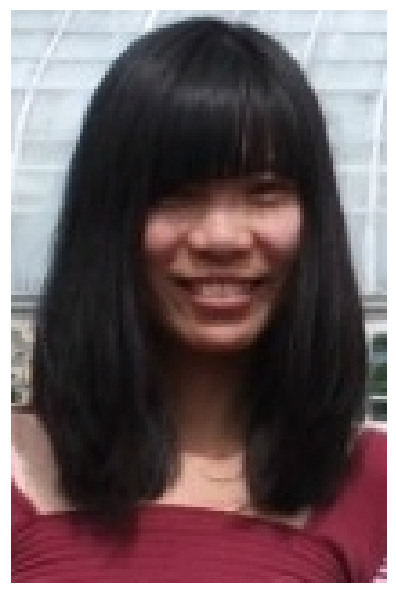

Lei Pan is a Corporation Internet Solution Developer at Nationwide Mutual Insurance Company in Columbus, Ohio. She received her Master of Science in Computer Science and Master of Business Administration from Bowling Green State University (BGSU) in December 2013. She worked for the Information Technology Services (ITS) department while attending BGSU, where she developed a General Study Writing web application for the English writing program. She and team members built an Android app - RollOn - when she attended Columbus Startup Weekend in March 2013. Angel investors gave her team second prize for the app. She is currently working on enterprise web applications which are reachable from www.nationwide.com.

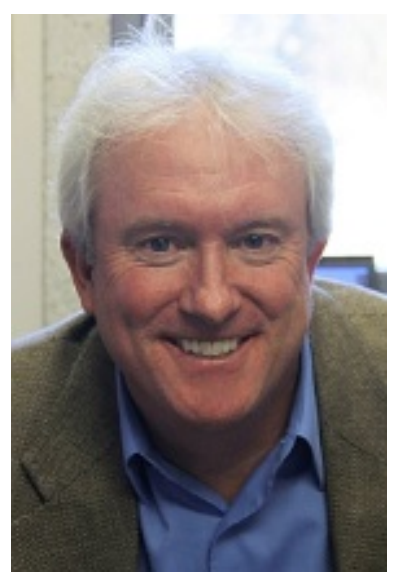

Dr. Kevin R. Parker is Chair and Professor of the Department of Informatics and Computer Science at Idaho State University. Parker's research interests include eGovernment and the elderly, business intelligence, and information assurance. He has published in such journals as International Journal of Business Intelligence Research, Education and Information Technologies, Informing Science, Journal of Information Systems Education, and Communications of the AIS. Dr. Parker's teaching interests include web development technologies, programming languages, data structures, and database management systems. Dr. Parker holds a B.A. in Computer Science from the University of Texas at Austin (1982), an M.S. in Computer Science from Texas Tech University (1991), and a Ph.D. in Management Information Systems from Texas Tech University (1995). 